\title{
Illusory lower limb levitation and involuntary moving toes following herpes zoster myelitis
}

\author{
Clara de Andrés ${ }^{1}$, Silvia Sánchez-Ramón ${ }^{2,4}$, M Luisa Martínez-Barriga ${ }^{1}$, Yasmina El Berdey ${ }^{1}$, ulia \\ Romero $^{3}$
}

1. Departments of Neurology, Hospital General Universitario Gregorio Marañón, Madrid, Spain. 2. Immunology, Hospital General Universitario Gregorio Marañón, Madrid, Spain. 3. Neuroradiology, Hospital General Universitario Gregorio Marañón, Madrid, Spain. 4. Department of Clinical Immunology and IdISSC, Hospital Universitario Clínico San Carlos, Madrid, Spain.

Correspondence: Silvia Sánchez-Ramón. Address: Department of Clinical Immunology and IdISSC Hospital Universitario Clínico San Carlos, C/ Profesor Martín Lagos SN, 28040-Madrid, Spain. Email: ssramon@salud.madrid.org

Received: August 25, 2014

DOI : $10.5430 /$ crim.v2n1p52
Accepted: September 12, 2014 Online Published: October 28, 2014

URL: http://dx.doi.org/10.5430/crim.v2n1p52

\section{Abstract}

We describe herein the first case report of illusory perception of levitation associated with VZV myelitis in a 68-year-old woman without known immune deficiency. She had absolutely no sensibility regarding position and movements of her lower limbs, while simultaneously she described spontaneous and continuous feeling as if her lower limbs floated in the air (levitation). Moreover, she intermittently presented involuntary bilateral spontaneously irregular slow-up oscillating painless movements, affecting ankles and toes. We discuss on the current pathogenetic hypotheses on both phenomena (levitation and involuntary limb movement) in the literature. Our patient represents a good example of the plastic aspects of the brain body schema with an anomalous positive disconnecting syndrome secondary to spinal cord lesion.

\section{Keywords}

Levitation, Involuntary moving toes, Varicella zoster virus, Myelitis

\section{I ntroduction}

Myelitis is an uncommon neurological complication of varicella-zoster virus (VZV) infection ${ }^{[1]}$ that usually develops in the immunocompromised host. It results from reactivation of latent infection at sensory ganglion neurons and posterior nerves roots. Clinical symptoms are subacute, beginning after days to weeks from the occurrence of typical skin rash and at the same level. However, the neurological involvement may also precede or even occur in the absence of rash. The spinal symptoms usually begin unilaterally or, if bilateral, were asymmetric. Neurological symptoms and sequels depend on the location of the lesion and on the severity of the underlying lesions. Spinal symptoms usually begin unilaterally or, if bilateral, are asymmetric, and may include autonomous, motor and sensory disorders either in isolation or in combination.

The feeling of body and/or limbs floating or raising in the air, namely levitation, is an unusual illusory sensations of position perception that may occur in peripheral or in central nervous system (CNS) lesions. We present a 67 year-old woman who developed lower limbs levitation and involuntary moving toes at onset of VZV myelitis. To our knowledge, this is the first report of illusory levitation associated with VZV myelitis. 


\section{Case presentation}

A 68-year-old Caucasian woman was first seen in January, 2005 with a 3-day history of severe propioceptive ataxia, numbness in hands, and oppressive submammary feeling, weakness in lower limbs and sphincters' dysfunction. During admission, the patient experienced vivid, spontaneous and continuous sensation as whether her lower half body were raising and floating in the air. At times, she complained of feeling as if her left leg were crossing over the right one. She was unstable on standing and unable to walk. Neurological examination revealed a cervico-thoracic C6-D4 level for all sensory modalities, loss of position sense on the passive movements in feet, ankles and hips, and moderate proximal lower limbs' weakness. Deep tendon reflexes were depressed with bilateral extensor plantar responses. Sphincters' function was loss. Bilateral spontaneous irregular slow-up oscillating painless movements affecting ankles and toes were intermittently present, which she was not able to suppress voluntarily. Full blood counts, liver and renal function analyses, electrolytes, immunoglobulin levels and chest X-ray were normal. Rheumatoid factor, antinuclear, anti-neutrophil cytoplasmic and antiphospholipid antibodies were negative. Multiple cultures in blood and cerebrospinal fluid (CSF) did not yield pathogenic microorganisms. Results of an extensive work-up for infectious diseases were negative. Serum IgM antibodies for VZV were positive. CSF contained $36 \mathrm{WBC} / \mathrm{mm}^{3}$, 98\% lymphocytes, and $46.2 \mathrm{mg} / \mathrm{dl}$ protein. Cranial MRI was normal. On spinal MRI at presentation (see Figure 1), T2-weighted images demonstrated diffuse increased signal from C4 to T11, representing oedema. Somatosensory evoked potentials in lower limbs were abolished. One week after onset, we observed a vesicular rash along the right anterior second intercostal nerve distribution. Diagnosis of VZV myelitis was done and therapy with IV acyclovir for 10 days, resulting in moderate beneficial effect. A new MRI 14-days later showed a marked decrease of the abnormal signal intensity, although persisting increased signal from C4-C6 (see Figure 2A), and preferentially in the right side (see Figure 2B). When last seen in March 2005, the legs levitation's illusions persisted. She needed help with bed-to-chair transfers and was unable to walk.

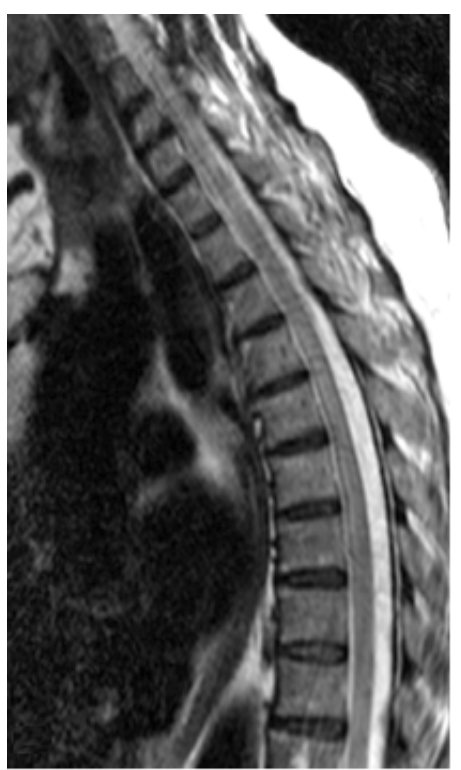

Figure 1. Spinal MRI at presentation (January, 2005). Sagittal T2-weighted images showed a marked diffuse hyperintense signal of the spinal cord from C4 to T11, representing oedema.
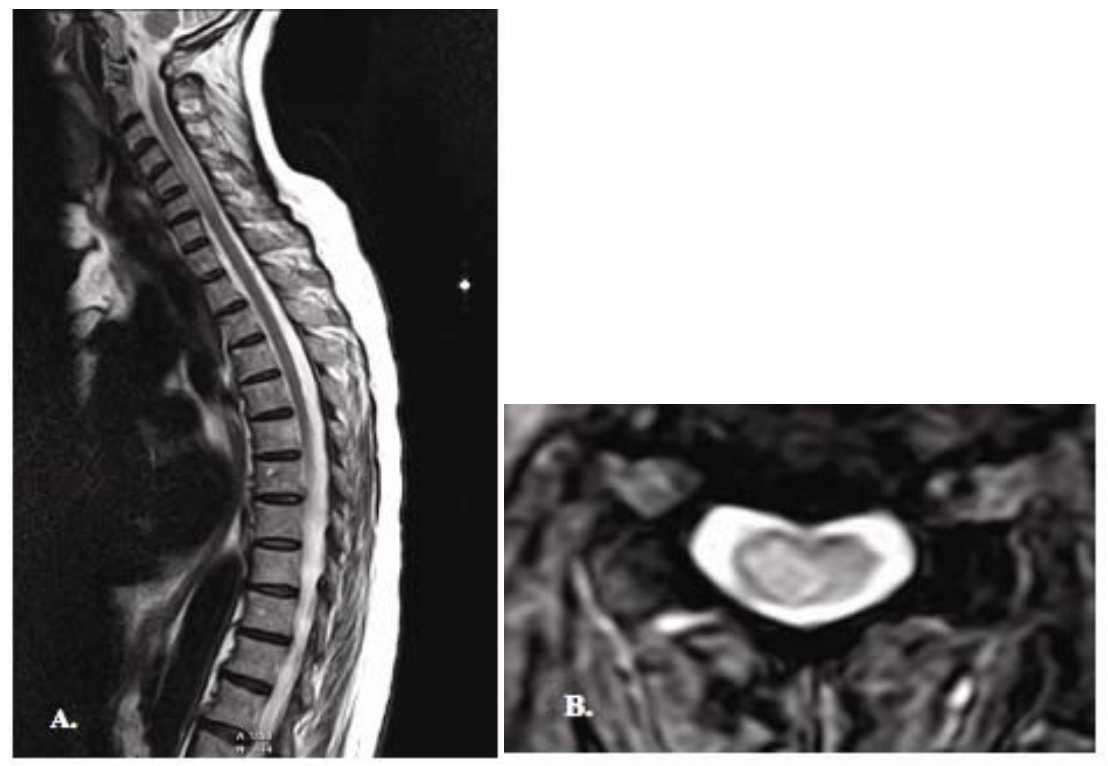

Figure 2. Second spinal MRI 2-weeks after admission (January, 2005). A) Sagittal T2-weighted images showed decrease in the hyperintense signal, although persisting from C4 to C6, representing oedema. B) Axial T2-weighted images at cervical level showed high signal intensity preferentially in the right spinal cord.

\section{Discussion}

We report the first description of the illusory perception of levitation associated with VZV myelitis. The diagnosis of VZV myelitis was made based on the basis of close relationship between the dorsal dermatome distribution of vesicular rash and 
the clinical symptoms and spinal cord level in the MRI and the positive serological test for VZV. VZV infects the spinal cord from contiguous affected dorsal root ganglia and nerves. Neuronal and glial direct infection, vasculitis and ischaemic necrosis, and immune-mediated demyelination have been proposed as mechanisms of its pathogenesis. Spinal MRI usually demonstrates multiple cervico-dorsal areas of $\mathrm{T} 2$ hyperintensities, affecting preferentially to the posterior columns of the spinal cord ${ }^{[2]}$. Our patient had absolutely no sensibility regarding position and movements of her lower limbs, including toes and ankles. Simultaneously, she described spontaneous and continuous feeling as if her legs were floating in the air, namely illusory levitation. Moreover, she intermittently presented involuntary bilateral spontaneously irregular slow-up oscillating painless movements, affecting ankles and toes. Both phenomena may result from lack of massive input to the brain, as consequence of spinal cord lesions. The mechanisms of both phenomena are not well known, and given that they may occur by different anatomic regions primary lesions, diverse pathogenetic hypothesis have been suggested. Patients with peripheral and CNS lesions (although more frequently in spinal cord), report perception distortions related position, shape, texture or temperature of the affected areas ${ }^{[3]}$. Paqueron $\mathrm{X}$ et al ${ }^{[4]}$ observed that during the course of regional, limbs, or spinal anaesthesia for orthopaedic surgery, the patients experienced perceptual alteration of body shape and were aware of its postural variations. Illusory perception of limb movement known as phantom phenomenon has been described in dorsal myelitis ${ }^{[5,6]}$. Ikeda $\mathrm{K}$ et al ${ }^{[7]}$ described phantom painful legs and moving toes syndrome 2-months after VZV myelitis. The primary VZV lesions were located at the posterior horn cells, probable involve the interneuron networks for the lower limbs, and the lesion in the posterior columns, as confirmed by MRI studies, may imply that the alterations of the ascending sensory afferents may be followed by deafferentation of the ventral thalamus and/or cortical somatosensory area for the lower limbs. They suggested that plasticity in the ascending somatosensory pathways including the posterior horn cells, probably involving the interneuron network, for the lower limbs may underlie the development of painful legs and moving toes syndrome. In some patients with right brain damage ${ }^{[8]}$, voluntary movements of the right hand induced a feeling of moving the contralesional hand while the subjects were looking themselves in the mirror, when conflicts among visual, somatic and motor information were maximal. The authors suggested that illusory movements are not generated in primary cortex, but are probably linked to higher disorder, at multimodal areas concerned with the integration of primary inputs into a unitary image of the body. In epileptic patients undergoing a presurgical investigation, bilateral feeling of levitation was elicited during electrode stimulation in the medial parietal region around the subparietal sulcus ${ }^{[9]}$. These data suggest that the medial parieto-occipital regions are closely linked to human visual motion processing system may be implicated.

We hypothesise that the lower-body levitation illusion and involuntary moving toes arise from central neural activities. The loss of sensory modalities increases the cortical representation of other sensory modalities and generates the reorganisation of new cortical synapses, which may in turn account of the failure to integrate the propioceptive, tactile and visual perception into her body image. Alternatively, certain post-injury alterations of behavior may reflect the collective action of synaptic connections that were present at time of injury, and may provide clues of the mechanisms underlying those behaviors ${ }^{[10]}$. In conclusion, our patient represents an example of the dynamic aspects of the brain body schema revealed by an anomalous positive (levitation and involuntary limb movement) disconnecting syndrome due to spinal cord lesion.

\section{References}

[1] Gilden D. Varicella zoster virus and central nervous nervous system syndromes. Herpes. 2004; 11 Suppl 2: 89A-94A. PMid:15319095

[2] Hirai T, Korogi Y, Hamatake S, et al. Varicella-zoster virus myelitis serial RM findings. Br J Radiol. 1996; 69: $1187-90$. PMid:9135480 http://dx.doi.org/10.1259/0007-1285-69-828-1187

[3] Nathan PW, Smith MC, Cook AW. Sensory affects in man of lesions of the posterior columns and of some other afferent pathways. Brain. 1986; 109: 1003-104. PMid:3096488 http://dx.doi.org/10.1093/brain/109.5.1003

[4] Paqueron X, Leguen M, Rosenthal D, et al. The phenomenology of body image distortions induced by regional anaesthesia. Brain. 2003; 126: 702-12. PMid:12566290 http://dx.doi.org/10.1093/brain/awg063 
[5] Sweet WH. "Phantom” sensations following intraspinal injury. Neurochirurgia (Stuttg). 1975; 18: 139-154.

[6] de Andrés C, Gil R, López E, et al. Nonpainful phantom sensation, illusory limb movement in a patient with dorsal myelitis. Neuropathological and SPECT finding. J Neurol. 2001; 248: 930-932.

[7] Ikeda K, et al. Painful legs and moving toes syndrome associated with herpes zoster myelitis. J Neurol Sci. $2004 ; 219: 147-50$. PMid:15050450 http://dx.doi.org/10.1016/j.jns.2004.01.011

[8] Zampini M, Moro V, Aglioti SM. Illusory movements of the contralesional hand in patients with body image disorders. J Neurol Neurosurg Psychiatry. 2004; 75: 1626-1628.

[9] Richer F, Martínez M, Robert M, et al. Stimulation of human somatosensory cortex: tactil and body displacement perceptions in medial regions. Exp Brain Res. 1993; 93: 173-6. PMid:8467887 http://dx.doi.org/10.1007/BF00227792

[10] Calancie B, Molano MR, Broton JG. Interlimb reflex and synaptic plasticity become evident months after human spinal cord injury. Brain. 2002; 125: 1150-6. PMid:11960903 http://dx.doi.org/10.1093/brain/awf114 